\title{
Higher Ring Derivation and Intuitionistic Fuzzy Stability
}

\author{
Ick-Soon Chang \\ Department of Mathematics, Mokwon University, Daejeon 302-729, Republic of Korea \\ Correspondence should be addressed to Ick-Soon Chang, ischang@mokwon.ac.kr
}

Received 3 May 2012; Accepted 12 June 2012

Academic Editor: Bing Xu

Copyright (C) 2012 Ick-Soon Chang. This is an open access article distributed under the Creative Commons Attribution License, which permits unrestricted use, distribution, and reproduction in any medium, provided the original work is properly cited.

We take account of the stability of higher ring derivation in intuitionistic fuzzy Banach algebra associated to the Jensen type functional equation. In addition, we deal with the superstability of higher ring derivation in intuitionistic fuzzy Banach algebra with unit.

\section{Introduction and Preliminaries}

The stability problem of functional equations has originally been formulated by Ulam [1]: under what condition does there exist a homomorphism near an approximate homomorphism? Hyers [2] answered the problem of Ulam under the assumption that the groups are Banach spaces. A generalized version of the theorem of Hyers for approximately additive mappings was given by Aoki [3] and for approximately linear mappings was presented by Rassias [4] by considering an unbounded Cauchy difference. The paper work of Rassias [4] has had a lot of influence in the development of what is called the generalized HyersUlam stability of functional equations. Since then, more generalizations and applications of the generalized Hyers-Ulam stability to a number of functional equations and mappings have been investigated (e.g., [5-7]). In particular, Badora [8] gave a generalization of the Bourgin's result [9], and he also dealt with the stability and the Bourgin-type superstability of derivations in [10]. Recently, fuzzy version is discussed in [11, 12]. Quite recently, the intuitionistic fuzzy stability problem for Jensen functional equation and cubic functional equation is considered in [13-15], respectively, while the idea of intuitionistic fuzzy normed space was introduced in [16], and there are some recent and important results which are directly related to the central theme of this paper, that is, intuitionistic fuzziness (see e.g., [17-20]). 
In this paper, we establish the stability of higher ring derivation in intuitionistic fuzzy Banach algebra associated to the Jensen type functional equation $l f(x+y / l)=f(x)+f(y)$. Moreover, we consider the superstability of higher ring derivation in intuitionistic fuzzy Banach algebra with unit.

We now recall some notations and basic definitions used in this paper.

Definition 1.1 (see [5]). Let $\mathcal{A}$ and $B$ be algebras over the real or complex field $\mathbb{F}$. Let $\mathbb{N}$ be the set of the natural numbers. From $m \in \mathbb{N} \cup\{0\}$, a sequence $H=\left\{h_{0}, h_{1}, \ldots, h_{m}\right\}$ (resp., $\left.H=\left\{h_{0}, h_{1}, \ldots, h_{k}, \ldots\right\}\right)$ of additive operators from $\mathcal{A}$ into $B$ is called a higher ring derivation of rank $m$ (resp., infinite rank) if the functional equation $h_{k}(x y)=\sum_{i=0}^{k} h_{i}(x) h_{k-i}(y)$ holds for each $k=0,1, \ldots, m$ (resp., $k=0,1, \ldots$ ) and for all $x, y \in \mathcal{A}$. A higher ring derivation $H$ of additive operators on $\mathcal{A}$, particularly, is called strong if $h_{0}$ is an identity operator.

Of course, a higher ring derivation of rank 0 from $\mathcal{A}$ into $B$ (resp., a strong higher ring derivation of rank 1 on $\mathbb{A}$ ) is a ring homomorphism (resp., a ring derivation). Note that a higher ring derivation is a generalization of both a ring homomorphism and a ring derivation.

Definition 1.2. A binary operation $*:[0,1] \times[0,1] \rightarrow[0,1]$ is said to be a continuous $t$-norm if it satisfies the following conditions:

(1) $*$ is associative and commutative, (2) $*$ is continuous, (3) $a * 1=a$ for all $a \in$ $[0,1]$, and (4) $a * b \leq c * d$ whenever $a \leq c$ and $b \leq d$ for each $a, b, c, d \in[0,1]$.

Definition 1.3. A binary operation $\diamond:[0,1] \times[0,1] \rightarrow[0,1]$ is said to be a continuous $t$-conorm if it satisfies the following conditions:

(1) $\diamond$ is associative and commutative, (2) $\diamond$ is continuous, (3) $a \diamond 0=a$ for all $a \in$ $[0,1]$, and (4) $a \diamond b \leq c \diamond d$ whenever $a \leq c$ and $b \leq d$ for each $a, b, c, d \in[0,1]$.

Using the notions of continuous t-norm and $t$-conorm, Saadati and Park [16] have recently introduced the concept of intuitionistic fuzzy normed space as follows.

Definition 1.4. The five-tuple $(\mathcal{X}, \mu, v, *, \diamond)$ is said to be an intuitionistic fuzzy normed space if $x$ is a vector space, $*$ is a continuous $t$-norm, $\diamond$ is a continuous $t$-conorm, and $\mu, v$ are fuzzy sets on $\mathcal{X} \times(0, \infty)$ satisfying the following conditions. For every $x, y \in \mathcal{X}$ and $s, t>0,(1) \mu(x, t)+$ $v(x, t) \leq 1,(2) \mu(x, t)>0$, (3) $\mu(x, t)=1$ if and only if $x=0$, (4) $\mu(\alpha x, t)=\mu(x, t /|\alpha|)$ for each $\alpha \neq 0$, (5) $\mu(x, t) * \mu(y, s) \leq \mu(x+y, t+s),(6) \mu(x, \cdot):(0, \infty) \rightarrow[0,1]$ is continuous, (7) $\lim _{t \rightarrow \infty} \mu(x, t)=1$ and $\lim _{t \rightarrow 0} \mu(x, t)=0$, (8) $v(x, t)<1$, (9) $v(x, t)=0$ if and only if $x=0,(10) v(\alpha x, t)=v(x, t /|\alpha|)$ for each $\alpha \neq 0,(11) v(x, t) \diamond \mu(y, s) \geq v(x+y, t+s),(12) v(x, \cdot)$ : $(0, \infty) \rightarrow[0,1]$ is continuous, (13) $\lim _{t \rightarrow \infty} v(x, t)=0$ and $\lim _{t \rightarrow 0} v(x, t)=1$.

In this case, $(\mu, v)$ is called an intuitionistic fuzzy norm.

Example 1.5. Let $(\mathcal{X},\|\cdot\|)$ be a normed space, $a * b=a b$, and $a \diamond b=\min \{a+b, 1\}$ for all $a, b \in[0,1]$. For all $x \in \mathcal{X}$ and every $t>0$, consider

$$
\mu(x, t)=\left\{\begin{array}{ll}
1, & \text { if } t>\|x\|, \\
0, & \text { if } t \leq\|x\|,
\end{array} \quad v(x, t)= \begin{cases}0, & \text { if } t>\|x\|, \\
1, & \text { if } t \leq\|x\|\end{cases}\right.
$$

Then $(x, \mu, v, *, \diamond)$ is an intuitionistic fuzzy normed space. 
Example 1.6. Let $(\mathcal{X},\|\cdot\|)$ be a normed space, $a * b=a b$, and $a \diamond b=\min \{a+b, 1\}$ for all $a, b \in[0,1]$. For all $x \in \mathcal{X}$ and every $t>0$ and $k=1,2$, consider

$$
\mu(x, t)=\left\{\begin{array}{ll}
\frac{t}{t+\|x\|}, & \text { if } t>0, \\
0, & \text { if } t \leq 0,
\end{array} \quad v(x, t)= \begin{cases}\frac{k\|x\|}{t+k\|x\|}, & \text { if } t>0, \\
0, & \text { if } t \leq 0 .\end{cases}\right.
$$

Then $(\mathcal{X}, \mu, v, *, \diamond)$ is an intuitionistic fuzzy normed space.

Definition 1.7 (see [21]). The five-tuple $(\mathcal{X}, \mu, v, *, \diamond)$ is said to be an intuitionistic fuzzy normed algebra if $\mathcal{X}$ is an algebra, $*$ is a continuous $t$-norm, $\diamond$ is a continuous $t$-conorm, and $\mu, v$ are fuzzy sets on $\mathcal{X} \times(0, \infty)$ satisfying the conditions (1)-(13) of the Definition 1.4. Furthermore, for every $x, y \in \mathcal{X}$ and $s, t>0,(14) \max \{\mu(x, t), \mu(y, s)\} \leq \mu(x y, t+$ $s),(15) \min \{v(x, t), v(y, s)\} \geq v(x y, t+s)$.

For an intuitionistic fuzzy normed algebra $(\mathcal{X}, \mu, v, *, \diamond)$, we further assume that (16) $a * a=a$ and $a \diamond a=a$ for all $a \in[0,1]$.

The concepts of convergence and Cauchy sequences in an intuitionistic fuzzy normed space are studied in [16]. Let $(\mathcal{X}, \mu, v, *, \diamond)$ be an intuitionistic fuzzy normed space or intuitionistic fuzzy normed algebra. A sequence $x=\left\{x_{k}\right\}$ is said to be intuitionistic fuzzy convergent to $L \in X$ if $\lim _{k \rightarrow \infty} \mu\left(x_{k}-L, t\right)=1$ and $\lim _{k \rightarrow \infty} \mathcal{v}\left(x_{k}-L, t\right)=0$ for all $t>0$. In this case, we write $(\mu, v)-\lim _{k \rightarrow \infty} x_{k}=L$ or $x_{k} \stackrel{I F}{\rightarrow} L$ as $k \rightarrow \infty$. A sequence $x=\left\{x_{k}\right\}$ in $(\mathcal{X}, \mu, v, *, \diamond)$ is said to be intuitionistic fuzzy Cauchy sequence if $\lim _{k \rightarrow \infty} \mu\left(x_{k+p}-x_{k}, t\right)=1$ and $\lim _{k \rightarrow \infty} \mathcal{v}\left(x_{k+p}-x_{k}, t\right)=0$ for all $t>0$ and $p=1,2, \ldots$. An intuitionistic fuzzy normed space (resp., intuitionistic fuzzy normed algebra) $(\mathcal{X}, \mu, v, *, \diamond)$ is said to be complete if every intuitionistic fuzzy Cauchy sequence in $(\mathcal{X}, \mu, v, *, \diamond)$ is intuitionistic fuzzy convergent in $(\mathcal{X}, \mu, v, *, \diamond)$. A complete intuitionistic fuzzy normed space (resp., intuitionistic fuzzy normed algebra) is also called an intuitionistic fuzzy Banach space (resp., intuitionistic fuzzy Banach algebra).

\section{Stability of Higher Ring Derivation in Intuitionistic Fuzzy Banach Algebra}

As a matter of convenience in this paper, we use the following abbreviation:

$$
\prod_{j=0}^{n} a_{j}:=a_{1} * a_{2} * \cdots * a_{n}, \quad \prod_{j=0}^{\infty} a_{j}:=a_{1} * a_{2} * \cdots
$$

In addition,

$$
\coprod_{j=0}^{n} a_{j}:=a_{1} \diamond a_{2} \diamond \cdots \diamond a_{n}, \quad \coprod_{j=0}^{\infty} a_{j}:=a_{1} \diamond a_{2} \diamond \cdots
$$

We begin with a generalized Hyers-Ulam theorem in intuitionistic fuzzy Banach space for the Jensen type functional equation. The following result is also the generalization of the theorem introduced in [13]. 
Theorem 2.1. Let $\mathbb{A}$ be a vector space, and let $f$ be a mapping from $\mathbb{A}$ to an intuitionistic fuzzy Banach space $(\boldsymbol{B}, \mu, v, *, \diamond)$ with $f(0)=0$. Suppose that $\varphi$ is a function from $\mathbb{A}$ to an intuitionistic fuzzy normed space $\left(C, \mu^{\prime}, v^{\prime}, *, \diamond\right)$ such that

$$
\begin{aligned}
& \mu\left(l f\left(\frac{x+y}{l}\right)-f(x)-f(y), t+s\right) \geq \mu^{\prime}(\varphi(x), t) * \mu^{\prime}(\varphi(y), s), \\
& v\left(l f\left(\frac{x+y}{l}\right)-f(x)-f(y), t+s\right) \leq v^{\prime}(\varphi(x), t) \diamond v^{\prime}(\varphi(y), s)
\end{aligned}
$$

for all $x, y \in \mathcal{A} \backslash\{0\}, t>0$ and $s>0$. If $l>1$ is a fixed integer, and $\varphi((l+1) x)=\alpha \varphi(x)$ for some real number $\alpha$ with $0<|\alpha|<l+1$, then there exists a unique additive mapping $\mathcal{L}: \mathcal{A} \rightarrow \mathbb{B}$ such that $\mathcal{L}(x):=(\mu, v)-\lim _{n \rightarrow \infty}\left(f\left((l+1)^{n} x\right) /(l+1)^{n}\right)$,

$$
\begin{aligned}
& \mu(\mathcal{L}(x)-f(x), t) \geq \prod_{j=0}^{\infty} M\left(x, \frac{((l+1)-\alpha) t}{2(l+1)}\right), \\
& v(\mathcal{L}(x)-f(x), t) \leq \coprod_{j=0}^{\infty} N\left(x, \frac{((l+1)-\alpha) t}{2(l+1)}\right)
\end{aligned}
$$

for all $x \in \mathcal{A}$ and $t>0$, where

$$
\begin{aligned}
& M(x, t):=\mu^{\prime}\left(\varphi(x), \frac{l+1}{4} t\right) * \mu^{\prime}\left(\varphi(-x), \frac{l+1}{4} t\right) * \mu^{\prime}\left(\varphi(-x), \frac{l+1}{4} t\right) * \mu^{\prime}\left(\varphi((l+1) x), \frac{l+1}{4} t\right), \\
& N(x, t):=v^{\prime}\left(\varphi(x), \frac{l+1}{4} t\right) \diamond v^{\prime}\left(\varphi(-x), \frac{l+1}{4} t\right) \diamond v^{\prime}\left(\varphi(-x), \frac{l+1}{4} t\right) \diamond v^{\prime}\left(\varphi((l+1) x), \frac{l+1}{4} t\right) .
\end{aligned}
$$

Proof. Without loss of generality, we assume that $0<\alpha<l+1$. From (2.3) and (2.4), we get

$$
\begin{gathered}
\mu(f(x)+f(-x), l t) \geq \mu^{\prime}\left(\varphi(x), \frac{l}{2} t\right) * \mu^{\prime}\left(\varphi(-x), \frac{l}{2} t\right) \\
v(f(x)+f(-x), l t) \leq v^{\prime}\left(\varphi(x), \frac{l}{2} t\right) \diamond\left(\varphi(-x), \frac{l}{2} t\right)
\end{gathered}
$$

for all $x \in \mathcal{A}$ and $t>0$. Again, by (2.3) and (2.4), we obtain

$$
\begin{aligned}
& \mu(l f(x)-f(-x)-f((l+1) x), l t) \geq \mu^{\prime}\left(\varphi(-x), \frac{l}{2} t\right) * \mu^{\prime}\left(\varphi((l+1) x), \frac{l}{2} t\right) \\
& v(l f(x)-f(-x)-f((l+1) x), l t) \leq v^{\prime}\left(\varphi(-x), \frac{l}{2} t\right) \diamond v^{\prime}\left(\varphi((l+1) x), \frac{l}{2} t\right)
\end{aligned}
$$


Abstract and Applied Analysis

5

for all $x \in \mathscr{A}$ and $t>0$. Combining (2.7) and (2.8), we arrive at

$$
\begin{aligned}
\mu((l+1) f(x)-f((l+1) x), 2 l t) \geq & \mu^{\prime}\left(\varphi(x), \frac{l}{2} t\right) * \mu^{\prime}\left(\varphi(-x), \frac{l}{2} t\right) * \mu^{\prime}\left(\varphi(-x), \frac{l}{2} t\right) \\
& * \mu^{\prime}\left(\varphi((l+1) x), \frac{l}{2} t\right), \\
v((l+1) f(x)-f((l+1) x), 2 l t) \leq & v^{\prime}\left(\varphi(x), \frac{l}{2} t\right) \diamond v^{\prime}\left(\varphi(-x), \frac{l}{2} t\right) \diamond v^{\prime}\left(\varphi(-x), \frac{l}{2} t\right) \\
& \diamond v^{\prime}\left(\varphi((l+1) x), \frac{l}{2} t\right),
\end{aligned}
$$

for all $x \in \mathcal{A}$ and $t>0$. This implies that

$$
\begin{aligned}
\mu\left(f(x)-\frac{f((l+1) x)}{(l+1)}, t\right) \geq & \mu^{\prime}\left(\varphi(x), \frac{l+1}{4} t\right) * \mu^{\prime}\left(\varphi(-x), \frac{l+1}{4} t\right) * \mu^{\prime}\left(\varphi(-x), \frac{l+1}{4} t\right) \\
& * \mu^{\prime}\left(\varphi((l+1) x), \frac{l+1}{4} t\right), \\
v\left(f(x)-\frac{f((l+1) x)}{(l+1)}, t\right) \leq & v^{\prime}\left(\varphi(x), \frac{l+1}{4} t\right) \diamond v^{\prime}\left(\varphi(-x), \frac{l+1}{4} t\right) \diamond v^{\prime}\left(\varphi(-x), \frac{l+1}{4} t\right) \\
& \diamond v^{\prime}\left(\varphi((l+1) x), \frac{l+1}{4} t\right),
\end{aligned}
$$

for all $x \in \mathcal{A}$ and $t>0$. Now we define

$$
\begin{aligned}
& M(x, t):=\mu^{\prime}\left(\varphi(x), \frac{l+1}{4} t\right) * \mu^{\prime}\left(\varphi(-x), \frac{l+1}{4} t\right) * \mu^{\prime}\left(\varphi(-x), \frac{l+1}{4} t\right) * \mu^{\prime}\left(\varphi((l+1) x), \frac{l+1}{4} t\right), \\
& N(x, t):=v^{\prime}\left(\varphi(x), \frac{l+1}{4} t\right) \diamond v^{\prime}\left(\varphi(-x), \frac{l+1}{4} t\right) \diamond v^{\prime}\left(\varphi(-x), \frac{l+1}{4} t\right) \diamond v^{\prime}\left(\varphi((l+1) x), \frac{l+1}{4} t\right),
\end{aligned}
$$

for all $x \in \mathcal{A}$ and $t>0$. Then we have by assumption

$$
M((l+1) x, t)=M\left(x, \frac{t}{\alpha}\right), \quad N((l+1) x, t)=N\left(x, \frac{t}{\alpha}\right),
$$

for all $x \in \mathcal{A}$ and $t>0$. Using (2.10) and (2.12), we get

$$
\begin{aligned}
\mu\left(\frac{f\left((l+1)^{n} x\right)}{(l+1)^{n}}-\frac{f\left((l+1)^{n+1} x\right)}{(l+1)^{n+1}}, \frac{\alpha^{n} t}{(l+1)^{n}}\right) & =\mu\left(f\left((l+1)^{n} x\right)-\frac{f\left((l+1)^{n+1} x\right)}{l+1}, \alpha^{n} t\right) \\
& \geq M\left((l+1)^{n} x, \alpha^{n} t\right)=M(x, t),
\end{aligned}
$$


6

Abstract and Applied Analysis

$$
\begin{aligned}
v\left(\frac{f\left((l+1)^{n} x\right)}{(l+1)^{n}}-\frac{f\left((l+1)^{n+1} x\right)}{(l+1)^{n+1}}, \frac{\alpha^{n} t}{(l+1)^{n}}\right) & =v\left(f\left((l+1)^{n} x\right)-\frac{f\left((l+1)^{n+1} x\right)}{l+1}, \alpha^{n} t\right) \\
& \leq N\left((l+1)^{n} x, \alpha^{n} t\right)=N(x, t),
\end{aligned}
$$

for all $x \in \mathcal{A}$ and $t>0$. Therefore, for all $n>m$, we have

$$
\begin{aligned}
& \mu\left(\frac{f\left((l+1)^{m} x\right)}{(l+1)^{m}}-\frac{f\left((l+1)^{n} x\right)}{(l+1)^{n}}, \sum_{j=m}^{n-1} \frac{\alpha^{j} t}{(l+1)^{j}}\right) \\
& \quad \mu\left(\sum_{j=m}^{n-1}\left[\frac{f\left((l+1)^{j} x\right)}{(l+1)^{j}}-\frac{f\left((l+1)^{j+1} x\right)}{(l+1)^{j+1}}\right], \sum_{j=m}^{n-1} \frac{\alpha^{j} t}{(l+1)^{j}}\right) \\
& \quad \prod_{j=m}^{n-1} \mu\left(\frac{f\left((l+1)^{j} x\right)}{(l+1)^{j}}-\frac{f\left((l+1)^{j+1} x\right)}{(l+1)^{j+1}}, \frac{\alpha^{j} t}{(l+1)^{j}}\right) \geq \prod_{j=m}^{n-1} M(x, t), \\
& v\left(\frac{f\left((l+1)^{m} x\right)}{\left.(l+1)^{m}-\frac{f\left((l+1)^{n} x\right)}{(l+1)^{n}}, \sum_{j=m}^{n-1} \frac{\alpha^{j} t}{(l+1)^{j}}\right)}\right. \\
& \quad=v\left(\sum_{j=m}^{n-1}\left[\frac{f\left((l+1)^{j} x\right)}{(l+1)^{j}}-\frac{f\left((l+1)^{j+1} x\right)}{(l+1)^{j+1}}\right], \sum_{j=m}^{n-1} \frac{\alpha^{j} t}{(l+1)^{j}}\right) \\
& \quad \leq \coprod_{j=m}^{n-1} v\left(\frac{f\left((l+1)^{j} x\right)}{(l+1)^{j}}-\frac{f\left((l+1)^{j+1} x\right)}{(l+1)^{j+1}}, \frac{\alpha^{j} t}{(l+1)^{j}}\right) \leq \coprod_{j=m}^{n-1} N(x, t),
\end{aligned}
$$

for all $x \in \mathcal{A}$ and $t>0$. Let $\varepsilon>0$ and $\delta>0$ be given. Since $\lim _{t \rightarrow \infty} \prod_{j=m}^{n-1} M(x, t)=1$ and $\lim _{t \rightarrow \infty} \coprod_{j=m}^{n-1} N(x, t)=0$, there exists some $t_{0}$ such that $\prod_{j=m}^{n-1} M\left(x, t_{0}\right)>1-\varepsilon, \bigsqcup_{j=m}^{n-1} N\left(x, t_{0}\right)<$ $\varepsilon$. Since $\sum_{j=0}^{\infty}\left(\alpha^{j} t /(l+1)^{j}\right)<\infty$, there exists a positive integer $n_{0}$ such that $\sum_{j=m}^{n-1}\left(\alpha^{j} t /(l+1)^{j}\right)<\delta$ for all $n>m \geq n_{0}$.

Then

$$
\begin{aligned}
\mu\left(\frac{f\left((l+1)^{m} x\right)}{(l+1)^{m}}-\frac{f\left((l+1)^{n} x\right)}{(l+1)^{n}}, \delta\right) & \geq \mu\left(\frac{f\left((l+1)^{m} x\right)}{(l+1)^{m}}-\frac{f\left((l+1)^{n} x\right)}{(l+1)^{n}}, \sum_{j=m}^{n-1} \frac{\alpha^{j} t_{0}}{(l+1)^{j}}\right) \\
& \geq \prod_{j=m}^{n-1} M\left(x, t_{0}\right)>1-\varepsilon,
\end{aligned}
$$


Abstract and Applied Analysis

$$
\begin{aligned}
v\left(\frac{f\left((l+1)^{m} x\right)}{(l+1)^{m}}-\frac{f\left((l+1)^{n} x\right)}{(l+1)^{n}}, \delta\right) & \leq v\left(\frac{f\left((l+1)^{m} x\right)}{(l+1)^{m}}-\frac{f\left((l+1)^{n} x\right)}{(l+1)^{n}}, \sum_{j=m}^{n-1} \frac{\alpha^{j} t_{0}}{(l+1)^{j}}\right) \\
& \leq \coprod_{j=m}^{n-1} N\left(x, t_{0}\right)<\varepsilon .
\end{aligned}
$$

This shows that $\left\{\left(f\left((l+1)^{n} x\right)\right) /\left((l+1)^{n}\right)\right\}$ is a Cauchy sequence in $\left(\mathbb{B}, \mu^{\prime}, \boldsymbol{v}^{\prime}, *, \diamond\right)$. Since $\boldsymbol{B}$ is complete, we can define a mapping $\mathcal{L}$ by $\mathcal{L}(x):=(\mu, v)-\lim _{n \rightarrow \infty}\left(f\left((l+1)^{n} x\right) /(l+1)^{n}\right)$ for all $x \in \mathcal{A}$. Moreover, if we let $m=0$ in (2.14), then we get

$$
\begin{aligned}
& \mu\left(\frac{f\left((l+1)^{n} x\right)}{(l+1)^{n}}-f(x), \sum_{j=0}^{n-1} \frac{\alpha^{j} t}{(l+1)^{j}}\right) \geq \prod_{j=0}^{n-1} M(x, t), \\
& \mathcal{v}\left(\frac{f\left((l+1)^{n} x\right)}{(l+1)^{n}}-f(x), \sum_{j=0}^{n-1} \frac{\alpha^{j} t}{(l+1)^{j}}\right) \leq \coprod_{j=0}^{n-1} N(x, t),
\end{aligned}
$$

for all $x \in \mathcal{A}$ and $t>0$. Therefore, we find that

$$
\begin{aligned}
& \mu\left(\frac{f\left((l+1)^{n} x\right)}{(l+1)^{n}}-f(x), t\right) \geq \prod_{j=0}^{n-1} M\left(x, \frac{t}{\sum_{j=0}^{n-1}\left(\alpha^{j} /(l+1)^{j}\right)}\right), \\
& v\left(\frac{f\left((l+1)^{n} x\right)}{(l+1)^{n}}-f(x), t\right) \leq \coprod_{j=0}^{n-1} N\left(x, \frac{t}{\sum_{j=0}^{n-1}\left(\alpha^{j} /(l+1)^{j}\right)}\right) .
\end{aligned}
$$

Next, we will show that $\mathcal{L}$ is additive mapping. Note that

$$
\begin{aligned}
& \mu\left(l \mathcal{L}\left(\frac{x+y}{l}\right)-\mathcal{L}(x)-\mathcal{L}(y), t\right) \geq \mu\left(l \mathcal{L}\left(\frac{x+y}{l}\right)-\frac{l f\left(\left((l+1)^{n}(x+y)\right) / l\right)}{(l+1)^{n}}, \frac{t}{4}\right) \\
& \quad * \mu\left(\frac{f\left((l+1)^{n} x\right)}{(l+1)^{n}}-\mathcal{L}(x), \frac{t}{4}\right) * \mu\left(\frac{f\left((l+1)^{n} y\right)}{(l+1)^{n}}-\mathcal{L}(y), \frac{t}{4}\right) \\
& \quad * \mu\left(\frac{l f\left(\left((l+1)^{n}(x+y)\right) /(l)\right)}{(l+1)^{n}}-\frac{f\left((l+1)^{n} x\right)}{(l+1)^{n}}-\frac{f\left((l+1)^{n} y\right)}{(l+1)^{n}}, \frac{t}{4}\right),
\end{aligned}
$$




$$
\begin{aligned}
& v\left(l \mathcal{L}\left(\frac{x+y}{l}\right)-\mathcal{L}(x)-\mathcal{L}(y), t\right) \leq v\left(l \mathcal{v}\left(\frac{x+y}{l}\right)-\frac{l f\left(\left((l+1)^{n}(x+y)\right) / l\right)}{(l+1)^{n}}, \frac{t}{4}\right) \\
& \diamond v\left(\frac{f\left((l+1)^{n} x\right)}{(l+1)^{n}}-\mathcal{L}(x), \frac{t}{4}\right) \diamond \mathcal{v}\left(\frac{f\left((l+1)^{n} y\right)}{(l+1)^{n}}-\mathcal{L}(y), \frac{t}{4}\right) \\
& \quad \diamond v\left(\frac{l f\left(\left((l+1)^{n}(x+y)\right) /(l)\right)}{(l+1)^{n}}-\frac{f\left((l+1)^{n} x\right)}{(l+1)^{n}}-\frac{f\left((l+1)^{n} y\right)}{(l+1)^{n}}, \frac{t}{4}\right) .
\end{aligned}
$$

On the other hand, (2.3) and (2.4) give the following:

$$
\begin{gathered}
\mu\left(\frac{l f\left(\left((l+1)^{n}(x+y)\right) / l\right)}{(l+1)^{n}}-\frac{f\left((l+1)^{n} x\right)}{(l+1)^{n}}-\frac{f\left((l+1)^{n} y\right)}{(l+1)^{n}}, \frac{t}{4}\right) \\
\geq \mu^{\prime}\left(\varphi(x),\left(\frac{l+1}{\alpha}\right)^{n} \frac{t}{8}\right) * \mu^{\prime}\left(\varphi(y),\left(\frac{l+1}{\alpha}\right)^{n} \frac{t}{8}\right), \\
v\left(\frac{l f\left(\left((l+1)^{n}(x+y)\right) / l\right)}{(l+1)^{n}}-\frac{f\left((l+1)^{n} x\right)}{(l+1)^{n}}-\frac{f\left((l+1)^{n} y\right)}{(l+1)^{n}}, \frac{t}{4}\right) \\
\leq v^{\prime}\left(\varphi(x),\left(\frac{l+1}{\alpha}\right)^{n} \frac{t}{8}\right) \diamond v^{\prime}\left(\varphi(y),\left(\frac{l+1}{\alpha}\right)^{n} \frac{t}{8}\right) .
\end{gathered}
$$

Letting $n \rightarrow \infty$ in (2.18) and (2.19), we yield

$$
\mu\left(l \mathcal{L}\left(\frac{x+y}{l}\right)-\mathcal{L}(x)-\mathcal{L}(y), t\right)=1, \quad v\left(l \mathcal{L}\left(\frac{x+y}{l}\right)-\mathcal{L}(x)-\mathcal{L}(y), t\right)=0 .
$$

So we see that $\mathcal{L}$ is additive mapping.

Now, we approximate the difference between $f$ and $\mathcal{L}$ in an intuitionistic fuzzy sense. By (2.17), we get

$$
\begin{aligned}
\mu(\mathcal{L}(x)-f(x), t) & \geq \mu\left(\mathcal{L}(x)-\frac{f\left((l+1)^{n} x\right)}{(l+1)^{n}}, \frac{t}{2}\right) * \mu\left(\frac{f\left((l+1)^{n} x\right)}{(l+1)^{n}}-f(x), \frac{t}{2}\right) \\
& \geq \prod_{j=0}^{\infty} M\left(x, \frac{((l+1)-\alpha) t}{2(l+1)}\right), \\
v(\mathcal{L}(x)-f(x), t) & \leq v\left(\mathcal{\nu}(x)-\frac{f\left((l+1)^{n} x\right)}{(l+1)^{n}}, \frac{t}{2}\right) \diamond v\left(\frac{f\left((l+1)^{n} x\right)}{(l+1)^{n}}-f(x), \frac{t}{2}\right) \\
& \leq \coprod_{j=0}^{\infty} N\left(x, \frac{((l+1)-\alpha) t}{2(l+1)}\right),
\end{aligned}
$$

for all $x \in \mathcal{A}$ and $t>0$ and sufficiently large $n$. 
In order to prove the uniqueness of $\mathcal{L}$, we assume that $T$ is another additive mapping from $A$ to $B$, which satisfies the inequality (2.5). Then

$$
\begin{aligned}
\mu(\mathcal{L}(x)-T(x), t) & \geq \mu\left(\mathcal{L}(x)-f(x), \frac{t}{2}\right) * \mu\left(T(x)-f(x), \frac{t}{2}\right) \\
& \geq \prod_{j=0}^{\infty} M\left(x, \frac{((l+1)-\alpha) t}{4(l+1)}\right), \\
v(\mathcal{L}(x)-T(x), t) & \leq v\left(\mathcal{L}(x)-f(x), \frac{t}{2}\right) \diamond v\left(T(x)-f(x), \frac{t}{2}\right) \\
& \leq \coprod_{j=0}^{\infty} N\left(x, \frac{((l+1)-\alpha) t}{4(l+1)}\right),
\end{aligned}
$$

for all $x \in \mathcal{A}$ and $t>0$. Therefore, due to the additivity of $\mathcal{L}$ and $T$, we obtain that

$$
\begin{aligned}
\mu(\mathcal{L}(x)-T(x), t) & =\mu\left(\mathcal{L}\left((l+1)^{n} x\right)-T\left((l+1)^{n} x\right),(l+1)^{n} t\right) \\
& \geq \prod_{j=0}^{\infty} M\left(x,\left(\frac{l+1}{\alpha}\right)^{n} \frac{((l+1)-\alpha) t}{4(l+1)}\right), \\
\mathcal{v}(\mathcal{L}(x)-T(x), t) & =v\left(\mathcal{L}\left((l+1)^{n} x\right)-T\left((l+1)^{n} x\right),(l+1)^{n} t\right) \\
& \leq \coprod_{j=0}^{\infty} M\left(x,\left(\frac{l+1}{\alpha}\right)^{n} \frac{((l+1)-\alpha) t}{4(l+1)}\right) .
\end{aligned}
$$

Since $0<\alpha<l+1, \lim _{n \rightarrow \infty}((l+1) / \alpha)^{n}=\infty$, and we get

$$
\lim _{n \rightarrow \infty} M\left(x,\left(\frac{l+1}{\alpha}\right)^{n} \frac{((l+1)-\alpha) t}{4(l+1)}\right)=1, \quad \lim _{n \rightarrow \infty} N\left(x,\left(\frac{l+1}{\alpha}\right)^{n} \frac{((l+1)-\alpha) t}{4(l+1)}\right)=0,
$$

that is, $\mu(\mathcal{L}(x)-T(x), t)=1$ and $v(\mathcal{L}(x)-T(x), t)=0$ for all $x \in \mathcal{A}, t>0$. So $\mathcal{L}=T$, which completes the proof.

In particular, we can prove the preceding result for the case when $\alpha>l+1$. In this case, the mapping $\mathcal{L}(x):=(\mu, v)-\lim _{n \rightarrow \infty}(l+1)^{n} f\left((l+1)^{-n} x\right)$. We now establish a generalized Hyers-Ulam stability in intuitionistic fuzzy Banach algebra for the higher ring derivation.

Theorem 2.2. Let $\mathcal{A}$ be an algebra, and let $F=\left\{f_{0}, f_{1}, \ldots, f_{k}, \ldots\right\}$ be a sequence of mappings from $\mathcal{A}$ to an intuitionistic fuzzy Banach algebra $(\boldsymbol{B}, \mu, \nu, *, \diamond)$ with $f_{k}(0)=0$ for each $k=0,1, \ldots$. Suppose 
that $\varphi$ is a function from $\&$ to an intuitionistic fuzzy normed algebra $\left(C, \mu^{\prime}, v^{\prime}, *, \diamond\right)$ such that for each $k=0,1, \ldots$,

$$
\begin{aligned}
& \mu\left(l f_{k}\left(\frac{x+y}{l}\right)-f_{k}(x)-f_{k}(y), t+s\right) \geq \mu^{\prime}(\varphi(x), t) * \mu^{\prime}(\varphi(y), s) \\
& v\left(l f_{k}\left(\frac{x+y}{l}\right)-f_{k}(x)-f_{k}(y), t+s\right) \leq v^{\prime}(\varphi(x), t) \diamond v^{\prime}(\varphi(y), s)
\end{aligned}
$$

for all $x, y \in \mathcal{A} \backslash\{0\}, t>0$ and $s>0$, and that $\Phi$ is a function from $\mathcal{A}$ to an intuitionistic fuzzy normed space $\left(D, \mu^{\prime \prime}, \nu^{\prime \prime}, *, \diamond\right)$ such that for each $k=0,1, \ldots$,

$$
\begin{aligned}
& \mu\left(f_{k}(x y)-\sum_{i=0}^{k} f_{i}(x) f_{k-i}(y), t+s\right) \geq \max \left\{\mu^{\prime \prime}(\Phi(x), t), \mu^{\prime \prime}(\Phi(y), s)\right\} \\
& v\left(f_{k}(x y)-\sum_{i=0}^{k} f_{i}(x) f_{k-i}(y), t+s\right) \leq \min \left\{v^{\prime \prime}(\Phi(x), t), v^{\prime \prime}(\Phi(y), s)\right\}
\end{aligned}
$$

for all $x, y \in \mathcal{A}, t>0$, and $s>0$. If $l>1$ is a fixed integer, $\varphi((l+1) x)=\alpha \varphi(x)$, and $\Phi((l+1) x)=$ $\beta \Phi(x)$ for some real numbers $\alpha$ and $\beta$ with $0<|\alpha|<l+1$ and $0<|\beta|<l+1$, then there exists a unique higher ring derivation $H=\left\{\mathcal{L}_{0}, \mathcal{L}_{1}, \ldots, \mathcal{L}_{k}, \ldots\right\}$ of any rank such that for each $k=0,1, \ldots$,

$$
\begin{aligned}
& \mu\left(\mathcal{L}_{k}(x)-f_{k}(x), t\right) \geq M\left(x, \frac{((l+1)-\alpha) t}{2(l+1)}\right), \\
& v\left(\mathcal{L}_{k}(x)-f_{k}(x), t\right) \leq N\left(x, \frac{((l+1)-\alpha) t}{2(l+1)}\right),
\end{aligned}
$$

for all $x \in \mathcal{A}$ and $t>0$. In this case,

$$
\begin{aligned}
& M(x, t):=\mu^{\prime}\left(\varphi(x), \frac{l+1}{4} t\right) * \mu^{\prime}\left(\varphi(-x), \frac{l+1}{4} t\right) * \mu^{\prime}\left(\varphi((l+1) x), \frac{l+1}{4} t\right), \\
& N(x, t):=v^{\prime}\left(\varphi(x), \frac{l+1}{4} t\right) \diamond v^{\prime}\left(\varphi(-x), \frac{l+1}{4} t\right) \diamond v^{\prime}\left(\varphi((l+1) x), \frac{l+1}{4} t\right) .
\end{aligned}
$$

Moreover, the identity

$$
\sum_{i=0}^{k} \mathcal{L}_{i}(y)\left\{\mathcal{L}_{k-i}(y)-f_{k-i}(y)\right\}=0
$$

holds for each $k=0,1, \ldots$ and all $x, y \in \mathcal{A}$. 
Proof. It follows by Theorem 2.1 that for each $k=0,1, \ldots$ and all $x \in \mathcal{A}$, there exists a unique additive mapping $\mathcal{L}_{k}: \mathcal{A} \rightarrow B$ given by

$$
\mathcal{L}_{k}(x):=(\mu, v)-\lim _{n \rightarrow \infty} \frac{f_{k}\left((l+1)^{n} x\right)}{(l+1)^{n}}
$$

satisfying (2.27) since $\left(C, \mu^{\prime}, v^{\prime}, *, \diamond\right)$ is an intuitionistic fuzzy normed algebra.

Without loss of generality, we suppose that $0<\beta<l+1$. Now, we need to prove that the sequence $H=\left\{\mathcal{L}_{0}, \mathcal{L}_{1}, \ldots, \mathcal{L}_{k}, \ldots\right\}$ satisfies the identity $\mathcal{L}_{k}(x y)=\sum_{i=0}^{k} \mathcal{L}_{i}(x) \mathcal{L}_{k-i}(y)$ for each $k=0,1, \ldots$ and all $x \in \mathcal{A}$. It is observed that for each $k=0,1, \ldots$,

$$
\begin{aligned}
& \mu\left(\mathcal{L}_{k}(x y)-\sum_{i=0}^{k} \mathcal{L}_{i}(x) f_{k-i}(y), t\right) \\
& \geq \mu\left(\mathcal{L}_{k}(x y)-\frac{f_{k}\left((l+1)^{n} x y\right)}{(l+1)^{n}}, \frac{t}{3}\right) * \mu\left(\frac{f_{k}\left((l+1)^{n} x y\right)}{(l+1)^{n}}-\sum_{i=0}^{k} \frac{f_{i}\left((l+1)^{n} x\right)}{(l+1)^{n}} f_{k-i}(y), \frac{t}{3}\right) \\
& \quad * \mu\left(\sum_{i=0}^{k} \frac{f_{i}\left((l+1)^{n} x\right)}{(l+1)^{n}} f_{k-i}(y)-\sum_{i=0}^{k} \mathcal{L}_{i}(x) f_{k-i}(y), \frac{t}{3}\right), \\
& v\left(\mathcal{L}_{k}(x y)-\sum_{i=0}^{k} \mathcal{L}_{i}(x) f_{k-i}(y), t\right) \\
& \leq v\left(\mathcal{L}_{k}(x y)-\frac{f_{k}\left((l+1)^{n} x y\right)}{(l+1)^{n}}, \frac{t}{3}\right) \diamond v\left(\frac{f_{k}\left((l+1)^{n} x y\right)}{(l+1)^{n}}-\sum_{i=0}^{k} \frac{f_{i}\left((l+1)^{n} x\right)}{(l+1)^{n}} f_{k-i}(y), \frac{t}{3}\right) \\
& \quad \diamond v\left(\sum_{i=0}^{k} \frac{f_{i}\left((l+1)^{n} x\right)}{(l+1)^{n}} f_{k-i}(y)-\sum_{i=0}^{k} \mathcal{L}_{i}(x) f_{k-i}(y), \frac{t}{3}\right)
\end{aligned}
$$

for all $x, y \in \mathcal{A}$ and $t>0$. On account of (2.26), we see that for each $k=0,1, \ldots$,

$$
\begin{aligned}
& \mu\left(\frac{f_{k}\left((l+1)^{n} x \cdot y\right)}{(l+1)^{n}}-\sum_{i=0}^{k} \frac{f_{i}\left((l+1)^{n} x\right)}{(l+1)^{n}} f_{k-i}(y), \frac{t}{3}\right) \\
& \quad=\mu\left(f_{k}\left((l+1)^{n} x \cdot y\right)-\sum_{i=0}^{k} f_{i}\left((l+1)^{n} x\right) f_{k-i}(y), \frac{(l+1)^{n} t}{3}\right) \\
& \quad \geq \max \left\{\mu^{\prime \prime}\left(\Phi(x),\left(\frac{l+1}{\beta}\right)^{n} \frac{t}{6}\right), \mu^{\prime \prime}\left(\Phi(y), \frac{(l+1)^{n} t}{6}\right)\right\}
\end{aligned}
$$




$$
\begin{gathered}
v\left(\frac{f_{k}\left((l+1)^{n} x \cdot y\right)}{(l+1)^{n}}-\sum_{i=0}^{k} \frac{f_{i}\left((l+1)^{n} x\right)}{(l+1)^{n}} f_{k-i}(y), \frac{(l+1)^{n} t}{3}\right) \\
\quad=v\left(f_{k}\left((l+1)^{n} x \cdot y\right)-\sum_{i=0}^{k} f_{i}\left((l+1)^{n} x\right) f_{k-i}(y), \frac{(l+1)^{n} t}{3}\right) \\
\quad \leq \min \left\{\mu^{\prime \prime}\left(\Phi(x),\left(\frac{l+1}{\beta}\right)^{n} \frac{t}{6}\right), v^{\prime \prime}\left(\Phi(y), \frac{(l+1)^{n} t}{6}\right)\right\},
\end{gathered}
$$

for all $x, y \in \mathcal{A}$ and $t>0$. Due to additivity of $\mathcal{L}_{k}$, for each $k=0,1, \ldots$,

$$
\begin{aligned}
& \mu\left(\sum_{i=0}^{k} \frac{f_{i}\left((l+1)^{n} x\right)}{(l+1)^{n}} f_{k-i}(y)-\sum_{i=0}^{k} \mathcal{L}_{i}(x) f_{k-i}(y), \frac{t}{3}\right) \\
& \quad \geq \prod_{i=0}^{k} \mu\left(f_{i}\left((l+1)^{n} x\right) f_{k-i}(y)-(l+1)^{n} \mathcal{L}_{i}(x) f_{k-i}(y), \frac{(l+1)^{n} t}{3(k+1)}\right), \\
& v\left(\sum_{i=0}^{k} \frac{f_{i}\left((l+1)^{n} x\right)}{(l+1)^{n}} f_{k-i}(y)-\sum_{i=0}^{k} \mathcal{L}_{i}(x) f_{k-i}(y), \frac{t}{3}\right) \\
& \leq \coprod_{i=0}^{k} v\left(f_{i}\left((l+1)^{n} x\right) f_{k-i}(y)-(l+1)^{n} \mathcal{L}_{i}(x) f_{k-i}(y), \frac{(l+1)^{n} t}{3(k+1)}\right)
\end{aligned}
$$

for all $x, y \in \mathcal{A}$ and $t>0$. In addition, we feel that

$$
\begin{aligned}
& \mu\left(f_{i}\left((l+1)^{n} x\right) f_{k-i}(y)-(l+1)^{n} \mathcal{L}_{i}(x) f_{k-i}(y), \frac{(l+1)^{n} t}{3(k+1)}\right) \\
& \geq \max \left\{\mu\left(f_{i}\left((l+1)^{n} x\right)-(l+1)^{n} \mathcal{L}_{i}(x), \frac{(l+1)^{n} t}{6(k+1)}\right), \mu\left(f_{k-i}(y), \frac{(l+1)^{n} \mathrm{t}}{6(k+1)}\right)\right\}, \\
& v\left(f_{i}\left((l+1)^{n} x\right) f_{k-i}(y)-(l+1)^{n} \mathcal{L}_{i}(x) f_{k-i}(y), \frac{(l+1)^{n} t}{3(k+1)}\right) \\
& \quad \leq \min \left\{v\left(f_{i}\left((l+1)^{n} x\right)-(l+1)^{n} \mathcal{L}_{i}(x), \frac{(l+1)^{n} t}{6(k+1)}\right), v\left(f_{k-i}(y), \frac{(l+1)^{n} t}{6(k+1)}\right)\right\} .
\end{aligned}
$$

Letting $n \rightarrow \infty$ in (2.31), (2.32), (2.33), and (2.34), we get $\mu\left(\mathcal{L}_{k}(x y)-\sum_{i=0}^{k} \mathcal{L}_{i}(x) f_{k-i}(y), t\right)=1$ and $v\left(\mathcal{L}_{k}(x y)-\sum_{i=0}^{k} \mathcal{L}_{i}(x) f_{k-i}(y), t\right)=0$. This implies that

$$
\mathcal{L}_{k}(x y)=\sum_{i=0}^{k} \mathcal{L}_{i}(x) f_{k-i}(y)
$$

for each $k=0,1, \ldots$ and all $x, y \in \mathcal{A}$. 
Using additivity of $\mathcal{L}_{k}$ and (2.35), we find that

$$
(l+1)^{n} \sum_{i=0}^{k} \mathcal{L}_{i}(x) f_{k-i}(y)=\mathcal{L}_{k}\left((l+1)^{n} x \cdot y\right)=\mathcal{L}_{k}\left(x \cdot(l+1)^{n} y\right)=\sum_{i=0}^{k} \mathcal{L}(x) f\left((l+1)^{n} y\right) .
$$

So we obtain $\sum_{i=0}^{k} \mathcal{L}_{i}(x) f_{k-i}(y)=\sum_{i=0}^{k} \mathcal{L}_{i}(x)\left(f_{k-i}\left((l+1)^{n} y\right) /(l+1)^{n}\right)$. Hence for each $k=0$, $1, \ldots$,

$$
\begin{aligned}
& \mu\left(\sum_{i=0}^{k} \mathcal{L}_{i}(x) f_{k-i}(y)-\sum_{i=0}^{k} \mathcal{L}_{i}(x) \frac{f_{k-i}\left((l+1)^{n} y\right)}{(l+1)^{n}}, t\right)=1, \\
& \mathcal{v}\left(\sum_{i=0}^{k} \mathcal{L}_{i}(x) f_{k-i}(y)-\sum_{i=0}^{k} \mathcal{L}_{i}(x) \frac{f_{k-i}\left((l+1)^{n} y\right)}{(l+1)^{n}}, t\right)=0,
\end{aligned}
$$

for all $x, y \in \mathcal{A}$ and $t>0$. This relation yields that for each $k=0,1, \ldots$,

$$
\begin{aligned}
\mu\left(\sum_{i=0}^{k} \mathcal{L}_{i}(x) \mathcal{L}_{k-i}(y)-\sum_{i=0}^{k} \mathcal{L}_{i}(x) f_{k-i}(y), t\right) \\
\geq \mu\left(\sum_{i=0}^{k} \mathcal{L}_{i}(x) \mathcal{L}_{k-i}(y)-\sum_{i=0}^{k} \mathcal{L}_{i}(x) \frac{f_{k-i}\left((l+1)^{n} y\right)}{(l+1)^{n}}, \frac{t}{2}\right) \\
* \mu\left(\sum_{i=0}^{k} \mathcal{L}_{i}(x) \frac{f_{k-i}\left((l+1)^{n} y\right)}{(l+1)^{n}}-\sum_{i=0}^{k} \mathcal{L}_{i}(x) f_{k-i}(y), \frac{t}{2}\right) \\
\geq \prod_{i=0}^{k} \mu\left(\mathcal{L}_{i}(x) \mathcal{L}_{k-i}(y)-\mathcal{L}_{i}(x) \frac{f_{k-i}\left((l+1)^{n} y\right)}{(l+1)^{n}}, \frac{t}{2(k+1)}\right), \\
\mathcal{v}\left(\sum_{i=0}^{k} \mathcal{L}_{i}(x) \mathcal{L}_{k-i}(y)-\sum_{i=0}^{k} \mathcal{L}_{i}(x) f_{k-i}(y), t\right) \\
\leq \nu\left(\sum_{i=0}^{k} \mathcal{L}_{i}(x) \mathcal{L}_{k-i}(y)-\sum_{i=0}^{k} \mathcal{L}_{i}(x) \frac{f_{k-i}\left((l+1)^{n} y\right)}{(l+1)^{n}}, \frac{t}{2}\right) \\
\diamond v\left(\sum_{i=0}^{k} \mathcal{L}_{i}(x) \frac{f_{k-i}\left((l+1)^{n} y\right)}{(l+1)^{n}}-\sum_{i=0}^{k} \mathcal{L}_{i}(x) f_{k-i}(y), \frac{t}{2}\right) \\
\leq \coprod_{i=0}^{k} \mathcal{v}\left(\mathcal{L}_{i}(x) \mathcal{L}_{k-i}(y)-\mathcal{L}_{i}(x) \frac{f_{k-i}\left((l+1)^{n} y\right)}{(l+1)^{n}}, \frac{t}{2(k+1)}\right),
\end{aligned}
$$


for all $x, y \in \mathcal{A}$ and $t>0$. On the other hand, we see that

$$
\begin{aligned}
& \mu\left(\mathcal{L}_{i}(x) \mathcal{L}_{k-i}(y)-\mathcal{L}_{i}(x) \frac{f_{k-i}\left((l+1)^{n} y\right)}{(l+1)^{n}}, \frac{t}{2(k+1)}\right) \\
& \geq \max \left\{\mu\left(\mathcal{L}_{i}(x), \frac{(l+1)^{n} t}{4(k+1)}\right), \mu\left(\mathcal{L}_{k-i}(y)-\frac{f_{k-i}\left((l+1)^{n} y\right)}{(l+1)^{n}}, \frac{t}{4(k+1)}\right)\right\} \\
& \mu\left(\mathcal{L}_{i}(x) \mathcal{L}_{k-i}(y)-\mathcal{L}_{i}(x) \frac{f_{k-i}\left((l+1)^{n} y\right)}{(l+1)^{n}}, \frac{t}{2(k+1)}\right) \\
& \quad \leq \min \left\{v\left(\mathcal{L}_{i}(x), \frac{(l+1)^{n} t}{4(k+1)}\right), v\left(\mathcal{L}_{k-i}(y)-\frac{f_{k-i}\left((l+1)^{n} y\right)}{(l+1)^{n}}, \frac{t}{4(k+1)}\right)\right\} .
\end{aligned}
$$

Sending $n \rightarrow \infty$ in (2.38) and (2.40), we have that for each $k=0,1, \ldots$,

$$
\begin{aligned}
& \mu\left(\sum_{i=0}^{k} \mathcal{L}_{i}(x) \mathcal{L}_{k-i}(y)-\sum_{i=0}^{k} \mathcal{L}_{i}(x) f_{k-i}(y), t\right)=1, \\
& \mathcal{v}\left(\sum_{i=0}^{k} \mathcal{L}_{i}(x) \mathcal{L}_{k-i}(y)-\sum_{i=0}^{k} \mathcal{L}_{i}(x) f_{k-i}(y), t\right)=0,
\end{aligned}
$$

for all $x, y \in \mathcal{A}$ and $t>0$. Thus, we conclude that

$$
\sum_{i=0}^{k} \mathcal{L}_{i}(x) \mathcal{L}_{k-i}(y)=\sum_{i=0}^{k} \mathcal{L}_{i}(x) f_{k-i}(y)
$$

for each $k=0,1, \ldots$ and all $x, y \in \mathcal{A}$.

Therefore, by combining (2.35) and (2.42), we get the required result, which completes the proof.

As a consequence of Theorem 2.2, we get the following superstability.

Corollary 2.3. Let $(\boldsymbol{B}, \mu, v, *, \diamond)$ be an intuitionistic fuzzy Banach algebra with unit, and let a sequence of operators $F=\left\{f_{0}, f_{1}, \ldots, f_{k}, \ldots\right\}$ on \& satisfy $f_{k}(0)=0$ for each $k=0,1, \ldots$, where $f_{0}$ is an identity operator. Suppose that $\varphi$ is a function from $A$ to an intuitionistic fuzzy normed algebra $\left(C, \mu^{\prime}, v^{\prime}, *, \diamond\right)$ satisfying (2.25) and (2.14) and that $\Phi$ is a function from $A$ to an intuitionistic fuzzy normed space $\left(D, \mu^{\prime \prime}, v^{\prime \prime}, *, \diamond\right)$ satisfying $(2.26)$. If $l>1$ is a fixed integer, $\varphi((l+1) x)=\alpha \varphi(x)$, and $\Phi((l+1) x)=\beta \Phi(x)$ for some real numbers $\alpha$ and $\beta$ with $0<|\alpha|<l+1$ and $0<|\beta|<l+1$, then $F$ is a strong higher ring derivation on $A$.

Proof. According to (2.30), we have $\mathcal{L}_{0}(x)=x$ for all $x \in \mathcal{A}$, and so $\mathcal{L}_{0}\left(=f_{0}\right)$ is an identity operator on $\mathcal{A}$. By induction, we get the conclusion. If $k=1$, then it follows from (2.29) that $f_{1}(\mathrm{x})=\mathcal{L}_{1}(x)$ holds for all $x \in \mathcal{A}$ since $\mathcal{A}$ contains the unit element. Let us assume that $f_{m}(x)=\mathcal{L}_{m}(x)$ is valid for all $x \in \mathcal{A}$ and $m<k$. Then (2.29) implies that $x\left\{\mathcal{L}_{m}(y)-f_{m}(y)\right\}=0$ for all $x, y \in \mathcal{A}$. Since $\mathcal{A}$ has the unit element, $f_{k}(y)=\mathcal{L}_{k}(y)$ for all $x \in \mathcal{A}$. Hence we conclude 
that $f_{k}(y)=\mathcal{L}_{k}(y)$ for each $k=0,1,2, \ldots$ and all $x \in \mathcal{A}$. So this tells us that $F$ is a higher ring derivation of any rank from $\mathcal{A}$ and $B$. The proof of the corollary is complete.

$\beta>l+1$.

We remark that we can prove the preceding result for the case when $\alpha>l+1$ and

\section{Acknowledgments}

The authors would like to thank the referees for giving useful suggestions and for the improvement of this paper. This work was supported by Basic Science Research Program through the National Research Foundation of Korea (NRF) funded by the Ministry of Education, Science and Technology (no. 2012-0002410).

\section{References}

[1] S. M. Ulam, A Collection of Mathematical Problems, Interscience Publishers, New York, NY, USA, 1960.

[2] D. H. Hyers, "On the stability of the linear functional equation," Proceedings of the National Academy of Sciences of the United States of America, vol. 27, pp. 222-224, 1941.

[3] T. Aoki, "On the stability of the linear transformation in Banach spaces," Journal of the Mathematical Society of Japan, vol. 2, pp. 64-66, 1950.

[4] T. M. Rassias, "On the stability of the linear mapping in Banach spaces," Proceedings of the American Mathematical Society, vol. 72, no. 2, pp. 297-300, 1978.

[5] Y.-S. Jung and I.-S. Chang, "On approximately higher ring derivations," Journal of Mathematical Analysis and Applications, vol. 343, no. 2, pp. 636-643, 2008.

[6] R. Saadati, Y. J. Cho, and J. Vahidi, "The stability of the quartic functional equation in various spaces," Computers \& Mathematics with Applications, vol. 60, no. 7, pp. 1994-2002, 2010.

[7] R. Saadati and C. Park, "Non-Archimedian L-fuzzy normed spaces and stability of functional equations," Computers \& Mathematics with Applications, vol. 60, no. 8, pp. 2488-2496, 2010.

[8] R. Badora, "On approximate ring homomorphisms," Journal of Mathematical Analysis and Applications, vol. 276, no. 2, pp. 589-597, 2002.

[9] D. G. Bourgin, "Approximately isometric and multiplicative transformations on continuous function rings," Duke Mathematical Journal, vol. 16, pp. 385-397, 1949.

[10] R. Badora, "On approximate derivations," Mathematical Inequalities \& Applications, vol. 9, no. 1, pp. 167-173, 2006.

[11] A. K. Mirmostafaee and M. S. Moslehian, "Fuzzy almost quadratic functions," Results in Mathematics, vol. 52, no. 1-2, pp. 161-177, 2008.

[12] A. K. Mirmostafaee and M. S. Moslehian, "Fuzzy versions of Hyers-Ulam-Rassias theorem," Fuzzy Sets and Systems, vol. 159, no. 6, pp. 720-729, 2008.

[13] S. A. Mohiuddine, "Stability of Jensen functional equation in intuitionistic fuzzy normed space," Chaos, Solitons E Fractals, vol. 42, no. 5, pp. 2989-2996, 2009.

[14] S. A. Mohiuddine, M. Cancan, and H. Şevli, "Intuitionistic fuzzy stability of a Jensen functional equation via fixed point technique," Mathematical and Computer Modelling, vol. 54, no. 9-10, pp. 24032409, 2011.

[15] M. Mursaleen and S. A. Mohiuddine, "On stability of a cubic functional equation in intuitionistic fuzzy normed spaces," Chaos, Solitons E Fractals, vol. 42, no. 5, pp. 2997-3005, 2009.

[16] R. Saadati and J. H. Park, "On the intuitionistic fuzzy topological spaces," Chaos, Solitons and Fractals, vol. 27, no. 2, pp. 331-344, 2006.

[17] M. Mursaleen, V. Karakaya, and S. A. Mohiuddine, "Schauder basis, separability, and approximation property in intuitionistic fuzzy normed space," Abstract and Applied Analysis, vol. 2010, Article ID 131868, 14 pages, 2010.

[18] M. Mursaleen and S. A. Mohiuddine, "Statistical convergence of double sequences in intuitionistic fuzzy normed spaces," Chaos, Solitons E Fractals, vol. 41, no. 5, pp. 2414-2421, 2009. 
[19] M. Mursaleen and S. A. Mohiuddine, "On lacunary statistical convergence with respect to the intuitionistic fuzzy normed space," Journal of Computational and Applied Mathematics, vol. 233, no. 2, pp. 142-149, 2009.

[20] M. Mursaleen, S. A. Mohiuddine, and O. H. H. Edely, “On the ideal convergence of double sequences in intuitionistic fuzzy normed spaces," Computers $\mathcal{E}$ Mathematics with Applications, vol. 59, no. 2, pp. 603-611, 2010.

[21] B. Dinda, T. K. Samanta, and U. K. Bera, "Intuitionistic fuzzy Banach algebra," Bulletin of Mathematical Analysis and Applications, vol. 3, no. 3, pp. 273-281, 2011. 


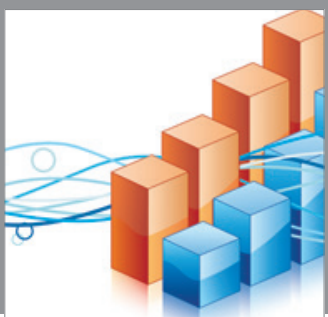

Advances in

Operations Research

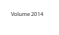

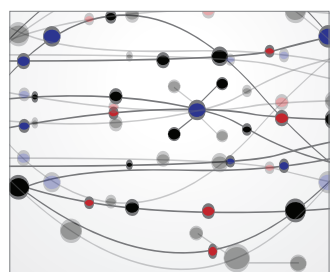

\section{The Scientific} World Journal
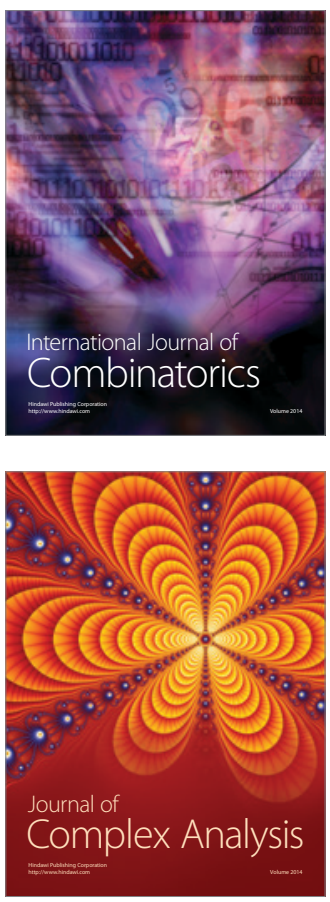

International Journal of

Mathematics and

Mathematical

Sciences
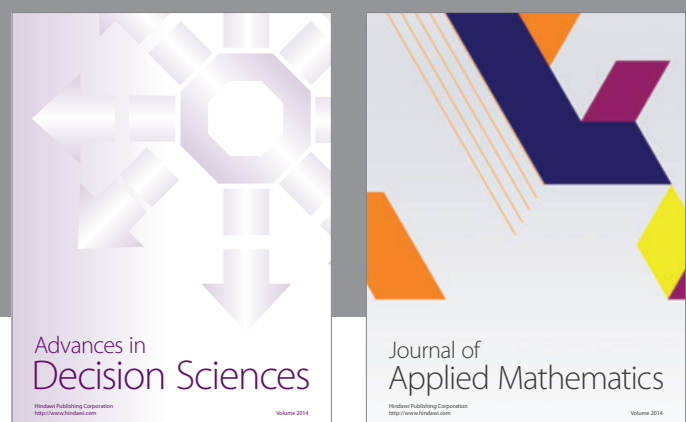

Journal of

Applied Mathematics
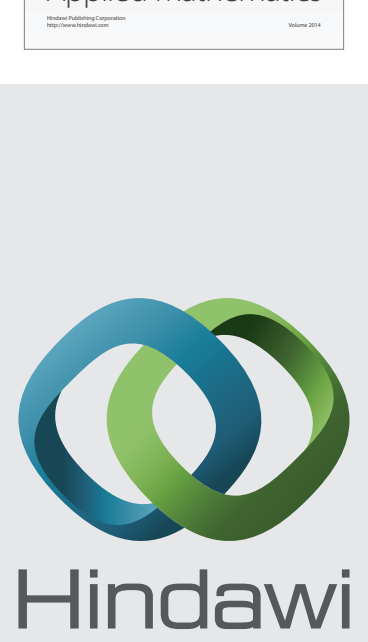

Submit your manuscripts at http://www.hindawi.com
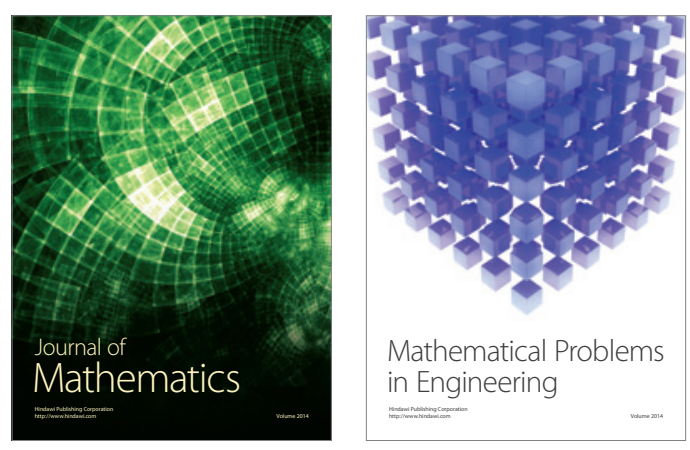

Mathematical Problems in Engineering
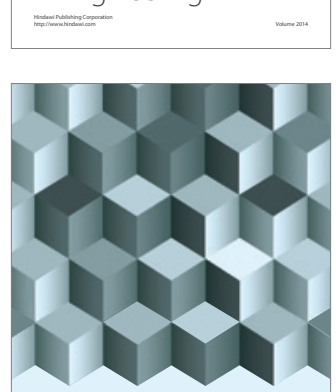

Journal of

Function Spaces
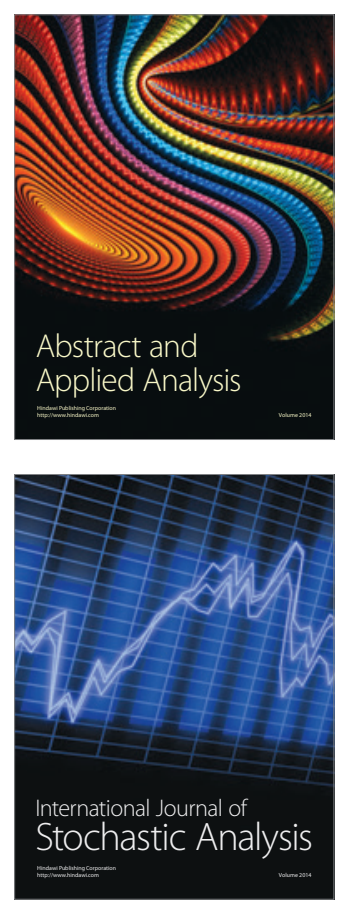

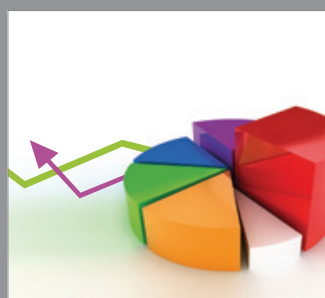

ournal of

Probability and Statistics

Promensencen
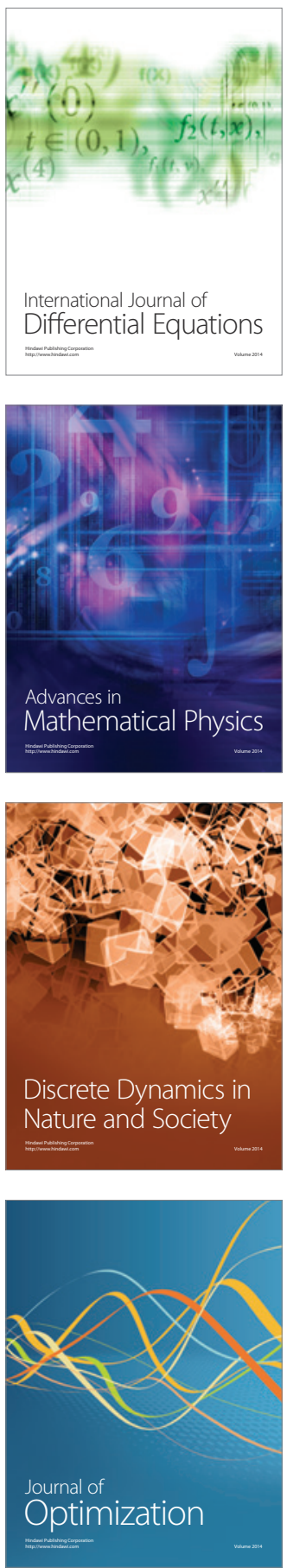